\title{
Genome Analysis of Enterobacter sp. LU1, a Highly Efficient Succinic Acid Producer
}

\author{
Hubert Szczerba ${ }^{1}$, Karolina Dudziak ${ }^{2}$, Zdzisław Targoński ${ }^{1}$ \\ ${ }^{1}$ Department of Biotechnology, Microbiology and Human Nutrition; University of Life Sciences in Lublin \\ 8 Skromna Street, 20-704 Lublin, Poland \\ hubert.szczerba@up.lublin.pl; karolina.dudziak@umlub.pl \\ ${ }^{2}$ Chair and Department of Biochemistry and Molecular Biology, Medical University of Lublin \\ 1 Chodźki Street, 20-093 Lublin, Poland \\ zdzislaw.targonski@up.lublin.pl
}

\section{Extended Abstract}

Succinic acid was recognized as one of the most important C4-building chemical platform that can be produce from side- and waste products and converted into high-valuable commodity and specialty chemicals. Until recently, succinic acid was produced mainly by chemical synthesis [1,2]. However, due to fluctuating oil prices and attempts to reduce environmental pollution, microbial synthesis of this metabolite has become a global point of interest. The number of microorganisms that have ability to overproduce succinic acid is still small. Moreover, most of the identified strains are already restricted by the ability to metabolize only simple carbon sources, such as glucose. Meantime, it is important to use microorganisms characterized by the efficient production of succinic acid on complex carbon sources, such as lactose and glycerol, which are often present in waste biomass.

In our previous study, the wild strain Enterobacter sp. LU1 has been isolated from goat rumen and identified. The strain has ability to overproduce succinic acid by utilization of cheap carbon sources such as waste glycerol and lactose. Physiological and biochemical properties of this strain have been determined. Preliminary studies have shown that higher level of succinic acid biosynthesis occurs in the presence of glycerol-lactose system than with glycerol, as the sole carbon source [3]. Lack of knowledge about molecular mechanisms that regulating the biosynthesis of fermentation products makes that performing of genetic characterization of this strain is necessary. Hence, this study is aimed at broadening the knowledge on basic genetic features of the Enterobacter sp. LU1 strain.

DNA isolation was performed and genome sequencing established by hybrid sequencing, including MinION ${ }^{\mathrm{TM}}$ paired with Illumina MiSeq platform was carried out. A single circular chromosome of 4,169,537-bp in size and one plasmid of 466,898-bp in size were obtained. A total of 4497 gene have been annotated in the genome of which 4323 were proteincoding genes. Subsequently, putative functions of coding DNA sequences (CDSs) were analyzed. Moreover, 25 rRNA, 84 tRNA, 5 ncRNA encoding genes and 60 pseudogenes were predicted. Additionally, two Clustered Regularly Interspaced Short Palindromic Repeats (CRISPR) assays were identified. Features such as antibiotic resistance, antibiotic production, bacteriocin production, presence of genomic islands were also determined.

This study was financially supported by the National Science Centre, Poland, within the Preludium 13 programme [Grant Number UMO-2017/25/N/NZ9/01736].

\section{References}

[1] N. Thuy, A. Kongkaev, A. Flood, “A Boontawan,” Bioresource Technology, vol. 233, pp. 342-352, 2017.

[2] Y. Zhao, W. Cao, Z. Wang, B. Zhang, K. Chen, "P. Ouyang,” Bioresource Technology, vol. 202, pp. 152-157, 2016.

[3] M. Podleśny, P. Jarocki, J. Wyrostek, T. Czernecki, J. Kucharska, A. Nowak, Z. Targoński, "Microbial biotechnology," vol 10, no. 2, pp. 492-501, 2016. 